\title{
A Multilevel Investigation of Fall Prevention Behavior Among Nursing Staff of South Korean Geriatric Hospitals
}

\author{
Yunhee Park ${ }^{1} \&$ Hyun-Jung Yun ${ }^{2}$ \\ ${ }^{1}$ Department of Nursing, College of Medicine, Wonkwang University, South Korea \\ ${ }^{2}$ Department of Nursing, Cheongju University, South Korea \\ Correspondence: Hyun-Jung Yun, Department of Nursing, Cheongju University, 298, Daesung-ro, Cheongju, \\ Choongbuk, 28503, South Korea. Tel: 82-43-229-7969. E-mail: hj0429@cju.ac.kr
}

Received: June 23, 2020 Accepted: July 22, 2020 Online Published: August 4, 2020

doi:10.5539/gjhs.v12n10p97

URL: https://doi.org/10.5539/gjhs.v12n10p97

\begin{abstract}
Background: There is lack of empirical evidence on whether organizational variables affect the fall prevention behavior of nursing staff working at Korean geriatric hospital. Aim This study aimed to investigate individual and organizational characteristics associated with the fall prevention behavior of nurses and nurse aides.

Methods: A descriptive cross-sectional research design was used. A convenient sample of 426 clinical nurses and nurse aides from 8 geriatric hospitals in South Korea was recruited between October and November 2019. Hierarchical regression analysis was used to estimate the effects of individual- and organization-level predictors.

Results: The result indicated that fall prevention self-efficacy $(\beta=0.41, p<.001)$ was a significant individual-level predictor. At the organizational level, Nurse to nurse aides ratio $(\beta=.21, p=.005)$ and number of patients per physical therapist $(\beta=-.28, p=.014)$ were significant predictors. Furthermore, there was a significant change of $\mathrm{R}^{2}$ $(p=.034)$ when organizational variables were included in the regression model.

Conclusion: To increase fall prevention behavior of nurse and nurse aides, administrators in geriatric hospital should recognize the importance of staffing, such as nurse and physical therapist. Further studies are proposed to investigate the empirical evidence about the association between organizational variables and patient outcomes.
\end{abstract}

Keywords: fall-prevention behavior, geriatric hospital, nurses, nurse aides, Organization

\section{Introduction}

\subsection{Background}

Due to the rapidly growing population of adults aged 65 or older in Korea, medical care costs for older people are also escalating dramatically (Korea Health Industry Development Institute [KHIDI], 2019). The people among the older adult population suffer from multiple chronic diseases and require long-term, complex treatment and care. The increased social responsibility for supporting this population has led to growing demands for geriatric hospitals that specialize in addressing their health problems (Park, 2020). As a result, geriatric hospitals have mushroomed in South Korea, now accounting for $46.2 \%$ of all hospitals, and the number is consistently on the rise year after year (KHIDI, 2019). As geriatric hospitals function as health care providers for patients in need of long-term treatment, the elderly population in South Korea relies on these hospitals to address their health problems and seek care supporting activities of daily living (ADL).

The most serious health threat to older residents of long-term care facilities is the risk of falling. Fall-induced injury can lead to longstanding pain, functional impairment, disability, and even death. Fear that is provoked among older adults who have experienced a fall manifests as increased social withdrawal and reduced engagement in ADL, ultimately causing severe depression and anxiety (Kannus, Sievänen, Palvanen, Järvinen, \& Parkkari, 2005). Therefore, registered nurses (RNs) and nurse aids (NAs), who are the primary care providers in geriatric hospitals, must be fully prepared for patient falls. Their preparation should be based on scientific evidence and meticulous training.

Numerous studies have generated fall prevention-related evidence (Kim \& Seo, 2017; Ju \& Jeon, 2018; Park, Ryu, Kwon, \& Lee, 2019). Additionally, academic societies and professional groups have developed guidelines to promote fall prevention behaviors (Chun et al., 2018; Kim et al., 2016); nevertheless, the prevalence of falls has 
not been markedly reduced. The global incidence of all falls in hospitals is still above $10 \%$ (Lim \& Gu, 2016), and falls are the leading cause of medical malpractice disputes in Korea (Korea Consumer Agency, 2017). This means that there is still a need for in-depth studies that examine factors related to falls.

Many past studies have identified fall prevention practice-related factors among RNs and NAs in geriatric hospitals, but most of them dealt with individual-level factors (Kim \& Seo 2017; Ju \& Jeon, 2018; Park et al., 2019). However, behaviors among members of an organization can be influenced by organizational traits and policies. This phenomenon is in line with the ecological model, which states that human behaviors are determined by the environment (McLeroy, Bibeau, Steckler, \& Glanz, 1988). Therefore, organizational characteristics, as well as individual characteristics, must be examined in order to modify individuals' behaviors in clinical practice.

Among various organizational characteristics, the nursing work environment is an indicator of whether the hospital fosters a supportive work environment for professional nurses (Lake, 2002). Therefore, a good nursing work environment is associated with higher work satisfaction among RNs in the organization, lower turnover intention, and less burnout (Chen \& Fang, 2016; Nantsupawat, Kunaviktikul, Nantsupawat, Wichaikhum, Thienthong, \& Poghosyan, 2017). These changes also improve patient outcomes. For example, researchers in South Korea found that hospitals whose RNs report high work satisfaction averaged $32 \%$ fewer reported incidences of inpatient falls than hospitals whose RNs report lower satisfaction (Cho, Chin, Kim, \& Hong, 2016). Hence, the nursing work environment is an essential factor to consider when examining predictors of fall prevention behaviors.

Furthermore, higher nursing staffing (ratio of nurses to patients) not only enhances the quality of nursing service but also has a positive impact on patients' health outcomes and nursing performance (Aiken, Clarke, Cheung, Sloane, \& Silber, 2003; Kim, Cho, June, Shin, \& Kim, 2012). The current RN to patient ratio in geriatric hospitals in Korea is $1: 6$, which means that one nurse is responsible for a relatively greater number of patients compared to their counterparts in acute care hospitals, which feature an RN-to-patient ratio of 1:2.5. Moreover, the Medical Service Act in South Korea allows for NAs to comprise two-thirds of organizations' required nursing staff. Therefore, geriatric hospitals feature extremely inadequate nursing staffing levels. This condition may influence the level of fall prevention activities; therefore, empirical evidence on the matter is needed.

\subsection{Aims}

The aim of this study was to identify the individual-level predictors of fall prevention practices of RNs and NAs in geriatric hospitals and to examine the actual effects of organization-level variables after controlling for the influence of the individual-level variables. The specific objectives are stated below:

1.2.1 Examine the participants' individual and organizational characteristics;

1.2.2 Examine the differences in fall prevention activities according to individual and organizational characteristics; and

1.2.3 Identify the predictors of fall prevention practices among the participants and examine the impact of organizational characteristics on fall prevention practice.

\section{Methods}

\subsection{Study Design}

This study utilized a descriptive survey designed to identify the individual and organizational predictors of fall prevention behaviors of RNs and NAs in geriatric hospitals.

\subsection{Study Population}

RNs and NAs working in eight private geriatric hospitals in J province, South Korea, were convenience sampled to participate in this study. As inclusion criteria, each participating nurse had to have a geriatric hospital career of one year or longer, and they had to sign, voluntarily, a statement of informed consent. A total of 50-100 questionnaires were distributed to each geriatric hospital in consideration of the size of their nursing staff and potential missing or careless responses, for a total of 520 questionnaires.

From the 520 questionnaires distributed, 436 were retrieved (83.8\%), with 21-96 participants in each hospital participating in the survey. After excluding 10 questionnaires with incomplete responses, data from 426 participants were used in the final analysis. 


\subsection{Study Instruments}

\subsubsection{Fall-Related Knowledge}

Fall-related knowledge was measured using an instrument developed by Kim (2002) and modified and adapted by Lee (2014). The total score for this 20 -item instrument ranges from $0-20$, and a higher score indicates a higher level of fall-related knowledge.

\subsubsection{Attitude Toward Falls}

Attitude toward falls was measured using an instrument developed by Kim (2002). This category comprises items about the nurse's level of interest and perceived importance of nursing activities for fall prevention. It consists of 13 items rated on a 5-point Likert scale. The total score ranges from 13-65, and a higher score indicates greater interest in and perceived importance of falls. The reliability of the tool, as measured with Cronbach's $\alpha$, was .95 in this study.

\subsubsection{Fall-Prevention Self-Efficacy}

Fall-prevention self-efficacy was measured using the instrument developed by Dykes et al. (2011) to measure fall-prevention self-efficacy in RNs and NAs and translated and validated by Uhm and Jeong (2014). This tool comprises 11 items about topics such as sharing of information for fall prevention and periodic use of a fall prevention assessment tool, with each item rated on a 6-point Likert scale from 1 "not at all confident" to 6 "completely confident"; total scores range from 11-66. The reliability of the tool as measured with Cronbach's $\alpha$ was .95 in this study.

\subsubsection{Fall-Prevention Behavior}

Fall-prevention behavior was measured using an instrument developed by Hwang (2011) for assessing the level of adherence to clinical care guidelines for falls. This 20-item tool employs a 5-point Likert scale; the scores are summed to generate a total score that ranges from 20-100. A higher score indicates higher adherence to fall prevention practice. The reliability of the tool, as measured with Cronbach's $\alpha$, was .91 in this study.

\subsubsection{Nursing Work Environment}

The nursing work environment was measured using the Korean version of the Practice Environment Scale of Nursing Work Index (PES-NWI), which was adapted by Cho et al. (2011) based on the tool originally developed by Lake (2002). This instrument comprises 29 items in five domains: nurses' participation in hospital affairs (9 items), nursing foundations for quality of care ( 9 items), nurse managers' ability, leadership, and support of nurses (4 items), staffing and resource adequacy (4 items), and collegial nurse-physician relations (3 items). Participants rate each item on a 4-point scale, from 1 "not at all true" to 4 "absolutely true." The total score ranges from 29-116, and a higher score indicates a better work environment for RNs. The reliability of the tool, as measured with Cronbach's $\alpha$, was .93 in the study by Cho et al. (2011) and .95 in this study. As this instrument is designed to be used with RNs, only the mean scores from RNs of each organization were applied toward the nursing work environment score for the corresponding organization.

\subsubsection{Organizational Characteristics}

Geriatric hospital grade, hospital beds, number of patients per nurse, number of patients per nursing staff (including both RNs and NAs), number of patients per doctor, and number of patients per physical therapist were obtained from the data posted on the Health Insurance Review and Assessment website (www.hira.or.kr). The nurse-to-nurse-assistant ratio was computed by comparing the number of patients per nurse to the number of patients per nursing staff based on the number of hospital beds. Hospital beds and number of patients per physical therapist induce multicollinearity when entered into the regression model, so they were converted to categorical variables. Geriatric hospital grade refers to a 5-level grade computed by calculating a standardized 5-point score for the nine categories for structure and 13 categories for care in each organization and weighting the scores. A lower grade number indicates a better rating.

\subsection{Data Collection and Ethical Consideration}

The instruments described above (section 2.3, subsections 2.3.1-6) were combined to create a self-administered questionnaire for the participants. Prior to executing the study, the validity of the study protocol and ethical considerations for the participants were reviewed by the appropriate institutional review board (WKIRB-201910-SB-080). We obtained permission for study participation from the director of each participating hospital. We also approached the heads of the nursing departments and provided them with an explanation of the study's purpose and procedures; we also asked for their cooperation in collecting data. 
Data were collected for approximately one month, from October to November 2019. The researchers visited the hospitals in -person and explained the study's purpose and methods to all potential participants. Prior to completing the questionnaire, the RNs and NAs who indicated a willingness to participate in the study were instructed to sign a written consent form and to submit the completed questionnaires, in sealed envelopes, to the nursing departments for the researchers to collect at a later time.

\subsection{Data Analysis}

The collected data were tested for normality to justify the use of parametric tests, and analyzed with descriptive statistics and univariate analysis using the STATA software package, version 13 (STATA, College Station, Texas). As organizational variables were highly correlated, the variance inflation factor (VIF) was computed to determine the presence of multicollinearity before entering them into the regression model. 8 hospitals did not meet the sample size required for multilevel analysis. Therefore, by assigning organization-level variables to each individual, it was treated as an individual-level variable and applied the ordinary least square (OLS) regression model. Hierarchical regression analysis was performed to estimate the effects of individual-level and organization-level predictors. First, each variable was analyzed with descriptive statistics, namely frequency with percentage and mean with standard deviation, and the variations of the dependent variables according to the participants' characteristics were analyzed using independent t-test and Pearson's correlation analysis. To identify the predictors of fall prevention behaviors, a hierarchical regression approach was used, where individual-level variables were first tested (model 1), after which the organization-level variables were added in step 2 to examine their effects while controlling for the effects of individual-level variables (model 2). Further, the proportions of variance explained before and after entering the organizational variables were compared and the statistical significance of the change in the proportion of variance explained $\left(\Delta \mathrm{R}^{2}\right)$ and $\mathrm{F}$ change $(\Delta \mathrm{F})$ using a partial F-test.

\section{Results}

\subsection{Subjects' Characteristics}

The mean age of the participants was $46.61( \pm 8.97)$ years, and $83.7 \%$ were married. A total of $76.3 \%$ of the participants stated that they have experienced patients' falls while working in the hospital. While $65.7 \%$ of the participants were NAs, $33.1 \%$ were staff nurses. Details by job are described at Table 1 .

Table 1. General characteristics of subjects $(\mathrm{N}=426)$

\begin{tabular}{|c|c|c|c|c|c|c|c|}
\hline \multirow{2}{*}{ Variables } & \multirow{2}{*}{ Categories } & \multicolumn{3}{|c|}{ Registered Nurse } & \multicolumn{3}{|l|}{ Nurse-aid } \\
\hline & & $\mathrm{n}(\%)$ & $\operatorname{Mean}(S D)$ & Median(Range) & $\mathrm{n}(\%)$ & $\operatorname{Mean}(S D)$ & Median(Range) \\
\hline Age (years) & & & $42.00(10.46)$ & $42(25,65)$ & & $49.01(6.92)$ & $50(24,63)$ \\
\hline \multirow[t]{3}{*}{ Marital status* } & Single & $38(27.5)$ & & & $26(9.3)$ & & \\
\hline & Married & $99(71.7)$ & & & $250(89.6)$ & & \\
\hline & Others & $1(0.7)$ & & & $3(1.1)$ & & \\
\hline \multirow{2}{*}{$\begin{array}{l}\text { Fall } \\
\text { experience* }\end{array}$} & Yes & $116(82.9)$ & & & $202(72.9)$ & & \\
\hline & No & $24(17.1)$ & & & $75(27.1)$ & & \\
\hline \multirow{2}{*}{$\begin{array}{l}\text { Work } \\
\text { experience } \\
\text { (months) }\end{array}$} & Total & & $158.49(108.76)$ & $132(1,411)$ & & $84.65(76.54)$ & $66(2,420)$ \\
\hline & Current job & & $37.87(40.03)$ & $30(1,300)$ & & $43.39(43.80)$ & $32(1,360)$ \\
\hline \multicolumn{2}{|c|}{ Work environment (1-4) } & & $2.79 \pm 0.15$ & $3(2,4)$ & & & \\
\hline
\end{tabular}

\subsection{Differences in Fall Prevention Behaviors According to Individual and Organizational Characteristics}

A comparison of the scores of RNs and NAs showed RNs had a significantly higher level of fall-related knowledge, while NAs had significantly higher fall prevention self-efficacy and fall prevention behaviors. There was no statistically significant difference in the attitude toward falls between RNs and NAs (Table 2). 
Table 2. Difference between nurses and nurse-assistants' fall-related factors on fall prevention behavior $(\mathrm{N}=426)$

\begin{tabular}{|c|c|c|c|c|}
\hline \multirow{2}{*}{ Variables } & \multirow{2}{*}{$\begin{array}{l}\text { Nurse } \\
M \pm S D\end{array}$} & Nurse-aid & \multirow{2}{*}{$t$} & \multirow[b]{2}{*}{$p$} \\
\hline & & $M \pm S D$ & & \\
\hline Fall-related knowledge (0-14) & $12.01 \pm 2.19$ & $10.93 \pm 2.28$ & 4.62 & $<<.001$ \\
\hline Attitude toward fall (13-65) & $46.12 \pm 4.06$ & $46.27 \pm 6.14$ & -0.25 & .801 \\
\hline Fall-prevention self-efficacy (11-66) & $44.06 \pm 5.48$ & $47.38 \pm 7.59$ & -5.10 & $<.001$ \\
\hline Fall-prevention behavior $(20-100)$ & $71.38 \pm 6.86$ & $74.99 \pm 6.23$ & -5.21 & $<.001$ \\
\hline
\end{tabular}

Regarding the correlations between individual and organizational characteristics and major variables, the individual-level variables that were significantly correlated with fall prevention behavior were attitude toward fall $(r=.13, p=.009)$ and fall prevention self-efficacy $(r=.43, p<.001)$. Organization-level variables that significantly correlated with fall prevention behavior were nurse to the nurse-assistant ratio $(r=.12, p=.013)$ and the number of patients per physical therapist $(r=-.12, p=.015)$ (Table 3$)$.

Table 3. Correlation between fall-prevention behavior and other variables ( $\mathrm{N}=426)$

\begin{tabular}{|c|c|c|c|c|c|c|c|c|c|c|}
\hline Variables & 1 & 2 & 3 & 4 & 5 & 6 & 7 & 8 & 9 & 10 \\
\hline
\end{tabular}

Individual level

1. Fall-related knowledge

1

2. Attitude toward fall $.22 * * * 1$

3. Fall-prevention self-efficacy

$-.04 \quad .12 * \quad 1$

Organizational level

4. Grade

5. Hospital beds

$\begin{array}{llll}.06 & .06 & .02 & 1\end{array}$

6. Number of patients per nursing staff

$\begin{array}{lllll}-.06 & -.04 & .06 & -.58 * * * & 1\end{array}$

7. Nurse to nurse-assistant ratio

$\begin{array}{llllll}-.03 & -.04 & .02 & -.30 * * * & .44 * * * & 1\end{array}$

8. Nurse work environment

$\begin{array}{lllllll}-.11 * & -.02 & .02 & -.34 * * * & .35 * * * & .52 * * * & 1\end{array}$

9. Number of patients per doctor

$\begin{array}{lllllllll}-.01 & -.03 & .02 & -.06 & .53 * * * & -.34 * * * & -.16 * * & 1\end{array}$

9. Number of patients per doctor

$\begin{array}{lllllllll}-.13 * * & -.04 & -.10 * & -.22 * * * & .10 * & -.12 * & -.18 * * * & -.16 * * & 1\end{array}$

10. Number of patients per physical therapist .05

$.03-.03$

.56

Dependent variable

11. Fall-prevention behavior

$-.05$

$.13 * * .43^{* * *}-.03$

.08

.07

$.12 * * \quad-.02-.12 *-.08$

${ }^{*} \mathrm{p}<0.05,{ }^{* *} \mathrm{p}<0.01,{ }^{* * *} \mathrm{p}<0.001$.

\subsection{Differences in Fall Prevention Behaviors According to Individual and Organizational Characteristics Predictors of Fall Prevention Behavior and Effects of Organization-Level Variables}

As there was a strong correlation among the organization-level independent variables, we examined whether the VIF of these variables was 10 or lower in order to eliminate the possibility of multicollinearity during regression analysis. In model 1 , which involved individual-level variables, attitude towards fall $(\beta=.09, p=.042)$, and fall-prevention self-efficacy $(\beta=.41, \mathrm{p}<.001)$ were identified as the predictors of fall-prevention behavior. In model 2, which estimated the effects of organization-level variables after controlling for the individual-level variables, the nurse to the nurse-assistant ratio $(\beta=.21, \mathrm{p}=.005)$, and the number of patients per physical therapist $(\beta=-.28, \mathrm{p}=.014)$ were identified as significant predictors. The individual-level variable of fall-prevention self-efficacy had significant effects even after the organization-level variables were entered $(\beta=.41, p<.001)$. While model 1, which only contained individual-level variables, explained $19.1 \%$ of the variance, the proportion 
of variance explained increased by $3 \%$ to $22.1 \%$ in model 2 with the added organization-level variables. This change was statistically significant $(\mathrm{p}=.034)$. These results suggest that organization-level variables must be considered when explaining fall prevention behaviors (Table 4).

Table 4. Factors affecting fall prevention behavior $(\mathrm{N}=426)$

\begin{tabular}{|c|c|c|c|c|c|}
\hline \multirow{2}{*}{ Variables } & & \multicolumn{2}{|c|}{ Model 1} & \multicolumn{2}{|c|}{ Model 2} \\
\hline & & $\beta$ & $p$ & $\beta$ & $p$ \\
\hline \multirow{3}{*}{$\begin{array}{l}\text { individual } \\
\text { level }\end{array}$} & Fall-related knowledge & -0.06 & .193 & -0.04 & .411 \\
\hline & Attitude toward fall & 0.09 & .042 & 0.08 & .066 \\
\hline & Fall-prevention self-efficacy & .41 & $<.001$ & 0.41 & $<.001$ \\
\hline \multirow{7}{*}{$\begin{array}{l}\text { Organizational } \\
\text { level }\end{array}$} & Grade & & & 0.10 & .156 \\
\hline & Hospital beds ${ }^{\dagger}$ & & & -0.06 & .642 \\
\hline & Number of patients per nursing staff & & & -0.16 & .087 \\
\hline & Nurse to nurse-assistant ratio & & & 0.21 & .005 \\
\hline & Number of patients per doctor & & & -0.01 & .840 \\
\hline & Number of patients per physical therapist ${ }^{\dagger}$ & & & -0.28 & .014 \\
\hline & Nurse work environment & & & -0.13 & .167 \\
\hline$F$ & & 32.838 & & 11.587 & \\
\hline$\Delta F$ & & & & 2.196 & \\
\hline$R^{2}$ & & .191 & & .221 & \\
\hline$\Delta R^{2}$ & & & & .030 & \\
\hline$p$ & & $<.001$ & & $<.001$ & \\
\hline
\end{tabular}

\section{Discussion}

This study aimed to identify the organizational predictors of fall prevention behaviors in RNs and NAs in geriatric hospitals in South Korea. As the results showed that organization-level variables have a significant impact on fall prevention behaviors, an approach that promotes fall prevention behaviors in RNs and NAs by improving organization-level variables could be useful in reducing falls among patients in geriatric hospitals.

Nurses are the "frontline" workers who provide direct care to patients in hospitals. Therefore, by monitoring patients' health outcomes and providing timely care, nurses serve as the first line of defense for minimizing adverse effects and events for patients. As fall prevention must be the primary approach to fall-related patient care, it essential for 24-hour bedside care to be available to older adult patients. However, geriatric wards in Korea allocate NAs and care assistants (who provide bedside assistance for patients' personal hygiene, eating, and moving) to provide much of the direct patient care. These assistants do not have the professional training and experience to provide the specialized and close care required to prevent falls (Park, 2020). Although there is no evidence for how to estimate the appropriate nursing personnel staffing for fall prevention, in a study conducted with RNs and NAs, researchers found that an inadequate nursing staffing level is a barrier to fall prevention (Park, 2020). In fact, a large number of patients per nurse was found to increase the risks for medication errors, pressure ulcers, and injurious falls (Cho et at., 2015). High workloads in geriatric hospitals also cause high turnover rates among RNs (Korean Hospital Nurses Association, 2012); therefore, ensuring adequate staffing levels that reduce nurses' workloads may be the most important measure for promoting patients' wellbeing and satisfaction. In this study, fall 
prevention behaviors increased with decreasing numbers of nursing staff, but the association was not statistically significant. The lack of variation sufficient to alter fall prevention behaviors is speculated to be attributed to the fact that geriatric hospitals in Korea only retain the minimum required staffing stipulated by the law. Further studies should examine the cost-effectiveness of increasing staff to produce long-term improvements in patient health outcomes in geriatric hospitals. Also, administrative lawmakers should utilize the results of such studies to provide policy direction regarding nursing personnel staffing levels.

When hiring nursing staff, it may be beneficial for geriatric hospitals to hire more RNs than NAs in terms of improving patients' health outcomes (Shin \& Hyun, 2015). The results of this study showed that RNs or NAs in organizations with a high nurse-to-nurse assistant ratio more frequently engaged in fall prevention behaviors ( $\beta$ $=.26, \mathrm{p}=.038$ ). This finding suggests that RNs play a crucial role in the nursing unit, which corroborates previous results indicating that falls among patients in geriatric hospitals decreased by $6.8 \%$ per 1 -hour increase in RNs' work hours (Shin \& Hyun, 2015). These results also support the argument that RNs contribute to improving patients' health outcomes.

Falls in elderly patients usually occur when they initiate movement, such as stepping down from their beds or getting into or out of an assistive device (Lim \& Gu, 2018). If there is a low proportion of RNs in the nursing staff, it is difficult to properly delegate nursing activities - such as moving patients or helping patients move by holding them - to NAs. However, without proper delegation, it may be difficult for NAs to recognize and perform essential nursing activities for patients independently. This is because Article 2-5 of the Medical Service Act in South Korea specifies that RNs must instruct NAs in their assistant work. Whereas RNs have the responsibility to accurately recognize the risk for a fall and make necessary decisions (Sung et al., 2007), NAs only passively perform tasks delegated by RNs. Consequently, NAs remain undertrained for independently identifying patients' problems and preparing for adverse events (Park, 2020). Therefore, under the current circumstances in Korea-where NAs are in charge of most of the direct care provided to patients - it is imperative that RNs instruct NAs to be aware of fall prevention behaviors as well as educating them about the need for fall prevention behaviors and specific protocol. Therefore, a work environment with an adequate number of RNs is more important than anything else, and increasing the proportion of RNs in the nursing staff is an essential requirement to improve patients' health outcomes (Cho et al., 2015; Kim \& Lee, 2015).

However, maintaining an appropriate nursing workforce to ensure high levels of good patient outcomes is a challenge for geriatric hospitals in Korea. According to one study, RNs working in 60 acute care hospitals in Korea are assigned nearly three times more patients per shift than their American nursing counterparts (Cho et al., 2015). While the mandatory RN to patient ratio in acute care hospitals in Korea is 1:2.5 (RNs in the hospital-to-number of patients), that in geriatric hospital is relatively low at 1:6, and two-thirds of the RNs in the geriatric hospitals can be replaced by NAs. However, it is challenging to maintain even this low nurse-to-patient ratio in Korea due to a growing shortage of nurses relative to the increasing demand for nursing services (Park, 2020). Therefore, to reduce falls for hospitalized older patients and improve overall healthcare for the aging population, it is necessary to improve the workforce composition. Specifically, while more NAs will be needed as the population continues to age, an even greater number of RNs are required to provide the level of specialized care necessary for fall prevention and improved outcomes for patients in geriatric hospitals.

Another organizational characteristics we examined is the nursing work environment. This characteristic is a particularly important indicator of the level of support for nursing professionals in clinical practice in a medical institution (Lake, 2002). A better nursing work environment refers to one with adequate RN staffing, appropriate rewards such as compliments and job promotions, and guaranteed RN involvement in administrative operations (Cho et al., 2011). A better nursing work environment leads to higher job satisfaction among RNs in an organization, with lower turnover intention and less burnout (Chen \& Fang, 2016; Nantsupawat, Kunaviktikul, Nantsupawat, Wichaikhum, Thienthong, \& Poghosyan, 2017). Moreover, a good nursing work environment has been reported to improve patient outcomes, such as reducing the incidences of bedsores by $39 \%$ and falls by $32 \%$ among inpatients; for this reason, the work environment is recognized as a major organization-level variable (Cho, Chin, Kim, \& Hong, 2015). However, in contrast to previous findings, our results indicate that the nursing work environment is not significantly associated with fall prevention behaviors $(\beta=-.13, p=.167)$.

Because this instrument commonly uses an aggregate of individual RN scores as an organization-level variable (Cho et at., 2015), we also generated the nursing work environment variable by measuring and averaging the scores among RNs and assigning that score to RNs and NAs in the corresponding organization. However, this instrument was developed to understand the characteristics of organizations comprising RNs, so we can speculate that it was not an appropriate tool to understand the context of geriatric hospitals, in which RNs and NAs 
collaborate in the work environment. Therefore, it may be difficult to propose a clear directional hypothesis pertaining to the relationship between nursing fall prevention behaviors and the work environment in geriatric hospitals based solely on our findings. The failure to use an appropriate tool suitable for the study population is a limitation of this study. Hence, subsequent studies using an instrument valid for nursing staff in geriatric hospitals are needed to clarify the relationship of the work environment to fall prevention in this setting.

It is known that patient outcomes improve when there are fewer patients per physician. Other researchers have demonstrated that a greater number of physicians working in a hospital brought about positive changes, such as decreasing patients' length of hospital stay and improving patients' rights (Lee, Ko, and Kim, 2011; Kim, \& Park, 2015). However, few studies have been conducted to examine the association of the number of physicians with nursing staff's adherence to care behaviors, making it difficult to anticipate the direction of the association between the two parameters. RNs in geriatric hospitals report patients' complaints to the physician and participate in the treatment decisions about the patient's state. However, due to the relatively low number of physicians in geriatric hospitals compared to acute care hospitals (KHIDI, 2019), RNs commonly feel psychological pressure from frequently having to perform duties that are normally the responsibility of a physician, such as wound dressing and tube insertion (Park \& Yeom, 2016). The current reality is that when only a small number of physicians are available, physicians cannot provide thorough care and RNs, based on their sense of duty to resolve patients' problems in a timely manner, fill in and perform physicians' roles themselves (Park \& Yeom, 2016). Considering the time spent performing physicians' duties, increasing the number of physicians in the hospital would allow RNs more time to engage in their own roles, including performing fall prevention behaviors. On the other hand, the results of this study revealed that fall prevention behaviors significantly increased when the number of patients per physical therapist decreased $(\beta=.28, p=.014)$, and physical therapists work independently (not with nurses). In the future, it is necessary to conduct empirical analyses to provide a specific understanding of how the percentage of other professionals on staff, such as physicians and physical therapists, influence the nurses' workloads and the impact of staff mix on patient outcomes.

Many studies have examined strategies that increase fall-related knowledge and self-efficacy influence attitudes about falls to promote fall prevention behaviors (Chang, Lee, \& Jung, 2019; Jung \& Jung, 2016; Kim \& Seo, 2017; Lim \& Gu, 2018). In the present study, attitude toward falls $(\beta=.09, \mathrm{p}=.042)$ and fall prevention self-efficacy ( $\beta$ $=.41, \mathrm{p}<.001)$ were identified in model 1 as significant predictors that only contained individual-level variables, and fall prevention self-efficacy remained significant in model 2 (covariate model) in which organization-level variables were entered $(\beta=.41, \mathrm{p}<.001)$. Fall-related knowledge was not significantly associated with fall prevention behaviors in our study, verifying results of previous studies of RNs (Jung \& Jung, 2016; Kim \& Seo, 2017). This result may be due to the healthcare institution accreditation system that was launched in 2010 in Korea that included fall prevention behaviors. This system prompted hospitals to administer thorough fall prevention education for their staff (Kim \& Seo, 2017). On the other hand, in the care of non-professional care assistants who were not given educational opportunities in hospital, the higher their knowledge, the higher their behavior for preventing fall ( $\mathrm{Lim} \& \mathrm{Gu}, 2018$ ). This evidence clearly supports knowledge as a prerequisite for action, and strategies to increase knowledge should not be neglected in the promotion of fall prevention behaviors among RNs and NAs.

In this study, RNs demonstrated a higher level of fall-related knowledge compared to NAs but less fall prevention behavior (Table 2). This contradiction appears to be due to the context of Korean geriatric wards in which RNs generally perform the roles of a charge nurse. Therefore, we can surmise that the knowledge parameter was not positively associated with fall prevention behaviors in this study because the NA group, which had a lower level of knowledge, showed a higher level of behavior. On the other hand, attitude toward falls and fall prevention self-efficacy were significantly associated with fall prevention behaviors, which was consistent with previous findings (Chang, Lee, \& Jung, 2019; Lim \& Gu, 2018). In particular, fall prevention self-efficacy was confirmed as a major predictor even after controlling for organization-level variables. Hence, strategies that increase self-efficacy should be considered when developing interventions to promote fall prevention behaviors in RNs and NAs.

In this study, several organization-level variables were taken into consideration to overcome the limitations of previous studies that attempted to explain fall prevention behaviors using only individual-level variables. However, it is also a limitation of this study that various variables other than staffing were not systemically considered. Nevertheless, the result demonstrating an increased $\mathrm{R}^{2}$ in the regression model in which organizational variables were entered after entering individual-level variables as covariates is highly significant, confirming that organizational characteristics significantly predict nursing staff's fall prevention behaviors in geriatric hospitals. Another limitation of the study, however, is that an OLS regression model used in which organization-level 
variables are entered with individual-level variables does not satisfy the statistical assumption for multilevel analysis due to the small number of hospitals studied $(n=8)$. Therefore, organization-level variation could not be rigorously estimated. Subsequent studies should expand the number of hospitals studied to estimate the valid effects of organization-level variables more accurately.

\section{Conclusions}

Given the marked escalation in the number of geriatric hospitals in South Korea in response to a rapidly aging population, in-depth assessments of the quality of care and patient outcome in geriatric hospitals are needed to inform the direction for proposed future changes. To promote fall prevention behaviors in RNs and NAs in geriatric hospitals, hospitals should consider implementing policies that increase the number of healthcare providers, such as RNs and physical therapists, in addition to those that increase fall prevention self-efficacy, an individual-level variable. The results of this study could be useful as baseline data for preventing falls in geriatric hospital patients and fostering a continuously safe environment. To obtain consistent evidence, large-scale studies should be conducted with more diverse geriatric hospitals for a valid examination of organization-level factors.

\section{Acknowledgements}

This study was supported by the research foundation of Wonkwang University, 2020.

\section{Competing Interests Statement}

The authors declare that there are no competing or potential conflicts of interest.

\section{References}

Aiken, L. H., Clarke, S. P., Cheung, R. B., Sloane, D. M., \& Silber, J. H. (2003). Educational levels of hospital nurses and surgical patient mortality. Jama, 290(12), 1617-1623. http://doi.org/10.1001/jama.290.12.1617

Chang, K. O., Lee, T. J., \& Jung, M. Y. (2019). The Effect of Knowledge, Attitude and Perceptions of Patient Safety Culture on Fall Prevention Activities in Mental Hospital Nurses. Journal of the Korea Academia-Industrial cooperation Society, 20(5), 372-383. https://doi.org/10.5762/KAIS.2019.20.5.372

Cho, E., Chin, D. L., Kim, S., \& Hong, O. (2016). The relationships of nurse staffing level and work environment with patient adverse events. Journal of Nursing Scholarship, 48(1), 74-82. https://doi.org/10.1111/jnu.12183

Cho, E., Choi, M., Kim, E. Y., Yoo, Y. I., \& Lee, N. J. (2011). Construct Validity and Reliability of the Korean Version of the Practice Environment Scale of Nursing Work Index for Korean Nurses. Journal of Korean Academy of Nursing, 41(3). https://doi.org/10.4040/jkan.2011.41.3.325

Chun, J. H., Kim, H. A., Kwak, M. J., Kim, H. S., Park, S. K., Kim, M. S., ... \& Kim, Y. S. (2018). Clinical Practice Guideline for Assessment and Prevention of Falls in Adult People. Quality Improvement in Health Care, 24(2), 41-61. https://doi.org/10.14371/QIH.2018.24.2.41

Dykes, P. C., Carroll, D., McColgan, K., Hurley, A. C., Lipsitz, S. R., Colombo, L., ... \& Middleton, B. (2011). Scales for assessing self-efficacy of nurses and assistants for preventing falls. Journal of advanced nursing, 67(2), 438-449. https://doi.org/10.1111/j.1365-2648.2010.05501.x

Eom, J. Y., \& Jung, D. Y. (2014). Psychometric test of Korean version of the self-efficacy for preventing (K-SEPF) fall scale among nurses who have worked in long-term care facilities and hospitals. Journal of Korean Gerontological Society, 34(3), 665-684. https://doi.org/10.1016/j.gerinurse.2016.10.012

Health insurance review \& assessment service. (2019). 2018 Nursing hospital evaluation report. Retrieved May 12 from http://www.hira.or.kr/re/diag/getDiagEvlList.do?pgmid=HIRAA030004000100

Hwang, I. Y. (2011). A study on knowledge, attitude, and implementation of clinical nursing practice guideline for fall prevention among hospital nurses working in the small and medium sized hospitals [dissertation]. Gyeongsang National University. Korea.

Ju, H. J., \& Jeon, M. Y. (2018). Effects of Walking Program with Dance on Gait, Cognition, and Risk of Falls of Elderly with Dementia in a Long-term Care Hospital. Journal of Korean Biological Nursing Science, 20(3), 141. https://doi.org/10.7586/jkbns.2018.20.3.141

Jung, J. Y., \& Jung, G. H. (2016). The Affect Factors of Geriatric Hospital Nurse's Falls Prevention Activities. Journal of Health Informatics and Statistics, 41(2), 203-211. https://doi.org/10.21032/jhis.2016.41.2.203

Kannus, P., Sievänen, H., Palvanen, M., Järvinen, T., \& Parkkari, J. (2005). Prevention of falls and consequent

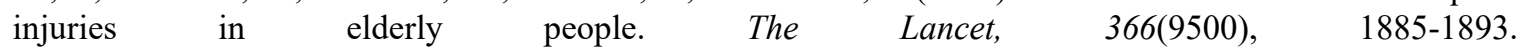
https://doi.org/10.1016/S0140-6736(05)67604-0 
Kim, B. H., \& Park, Y. S. (2015). Convalescent Hospital Doctors and Nursing Staff Awareness of the Differential Rating: Focused on Doctors, Nursing Staff. The Journal of the Korea Contents Association, 15(12), 285-293. https://doi.org/10.5392/JKCA.2015.15.12.285

Kim, C. G. (2003). An analysis of fall incidence rate and the related factors of fall in hospitalized patients. Unpublished master's thesis, Seoul National University, Seoul.

Kim, K. I., Jung, H. K., Kim, C. O., Kim, S. K., Cho, H. H., Kim, D. Y., ... \& Kim, H. J. (2016). Evidence-based guideline for fall prevention in Korea. Journal of the Korean Geriatrics Society, 20(1), 1-28. https://doi.org/10.4235/jkgs.2016.20.1.1

Kim, E. H., \& Lee, E. (2015). Nursing outcomes of inpatient on level of nursing staffing in long term care hospitals. Journal of the Korean Data and Information Science Society, 26(3), 715-727. https://doi.org/10.7465/jkdi.2015.26.3.715

Kim, S. H., \& Seo, J. M. (2017). Geriatric hospital nurses' knowledge, attitude toward falls, and fall prevention activities. Journal of Korean Gerontological Nursing, 19(2), 81-91. https://doi.org/10.17079/jkgn.2017.19.2.81

Kim, Y., Cho, S. H., June, K. J., Shin, S. A., \& Kim, J. (2012). Effects of Hospital Nurse Staffing on in-hospital Mortality, Pneumonia, Sepsis, and Urinary Tract Infection in Surgical Patients. Journal of Korean Academy of Nursing, 42(5). https://doi.org/10.4040/jkan.2012.42.5.719

Korea health industry development institute. (2019). Statistical results of the 2018 health industry. Retrieved May 12 from https://khiss.go.kr/board/view?menuId=MENU00305\&linkId=175433

Korean Hospital Nurses Association. (2012). Survey on the current nurse deployment. A report of hospital nurses association, 99-109. Seoul: Hospital Nurses Association.

Lake, E. T. (2002). Development of the practice environment scale of the Nursing Work Index. Research in nursing \& health, 25(3), 176-188. https://doi.org/10.1002/nur.10032

Lee, H., Ko, Y. K., \& Kim, M. W. (2011). The effects of medical staffing level on length of stay. Journal of Korean Academy of Nursing Administration, 17(3), 327-335. https://doi.org/10.11111/jkana.2011.17.3.327

Lim, J. O., \& Gu, M. O. (2018). The Influence of Fall-Related Knowledge and Fall Prevention Self-Efficacy of Care-Givers Working in Long-term Care Hospitals with Older Adults with Dementia on Fall Prevention Behaviors and Fall Management Behaviors. The Korean Journal of Health Service Management, 12(4), 155-172. https://doi.org/10.12811/kshsm.2018.12.4.172

McLeroy, K. R., Bibeau, D., Steckler, A., \& Glanz, K. (1988). An ecological perspective on health promotion programs. Health education quarterly, 15(4), 351-377. https://doi.org/10.1177/109019818801500401

Nantsupawat, A., Kunaviktikul, W., Nantsupawat, R., Wichaikhum, O. A., Thienthong, H., \& Poghosyan, L. (2017). Effects of nurse work environment on job dissatisfaction, burnout, intention to leave. International nursing review, 64(1), 91-98. https://doi.org/10.1111/inr.12342

Park, Y. (2020). Barriers to the practice of fall prevention behavior by nurses and nurse assistants at geriatric hospitals. Journal of the Korean Gerontological Society, 40(2), 311-323 https://doi.org/10.31888/JKGS.2020.40.2.311

Park, B. M., Ryu, H. S., Kwon, K. E., \& Lee, C. Y. (2019). Development and effect of a fall prevention program based on the King's Goal Attainment Theory for fall high-risk elderly patients in long-term care hospital. Journal of Korean Academy of Nursing, 49(2), 203-214. https://doi.org/10.4040/jkan.2019.49.2.203

Park, S., \& Yeom, E. Y. (2016). Experience of the role conflict of nurses in long-term care hospitals. The Journal of the Korea Contents Association, 16(7), 368-381. https://doi.org/10.5392/JKCA.2016.16.07.368

Shin, J. H., \& Hyun, T. K. (2015). Nurse staffing and quality of care of nursing home residents in Korea. Journal of Nursing Scholarship, 47(6), 555-564. https://doi.org/10.1111/jnu.12166

Sung, M. H., Kang, J. H., Shon, J. T., Yoo, S. Y., Lee, E. J., \& Jung, K. Y. (2007). Nursing process and critical thinking. Seoul: Jungmunkak.

\section{Copyrights}

Copyright for this article is retained by the author(s), with first publication rights granted to the journal.

This is an open-access article distributed under the terms and conditions of the Creative Commons Attribution license (http://creativecommons.org/licenses/by/4.0/). 